\title{
A hymenopterists' guide to the Hymenoptera Anatomy Ontology: utility, clarification, and future directions
}

Katja C. Seltmann ${ }^{1,2}$, Matthew J. Yoder ${ }^{1,3}$, István Mikó ${ }^{1,4}$, Mattias Forshage ${ }^{5}$, Matthew A. Bertone', Donat Agosti ${ }^{6,7}$, Andrew D. Austin ${ }^{8}$, James P. Balhoff', Marek L. Borowiec ${ }^{10}$, Seán G. Brady'1, Gavin R. Broad ${ }^{12}$, Denis J. Brothers ${ }^{13}$, Roger A. Burks ${ }^{14}$, Matthew L. Buffington ${ }^{15}$, Heather M. Campbell', Kelly J. Dew', Andrew F. Ernst', José L. Fernández-Triana ${ }^{16}$, Michael W. Gates ${ }^{15}$, Gary A.P. Gibson ${ }^{16}$, John T. Jennings ${ }^{8}$, Norman F. Johnson ${ }^{14}$, Dave Karlsson ${ }^{17}$, Ricardo Kawada ${ }^{18}$, Lars Krogmann ${ }^{19}$, Robert R. Kula ${ }^{15}$, Patricia L. Mullins', Michael Ohl20, Claus Rasmussen ${ }^{21}$, Fredrik Ronquist ${ }^{22}$, Susanne Schulmeister ${ }^{23}$, Michael J. Sharkey ${ }^{24}$, Elijah Talamas ${ }^{14}$, Erika Tucker ${ }^{24}$, Lars Vilhelmsen ${ }^{25}$, Philip S. Ward ${ }^{10}$, Robert A. Wharton ${ }^{26}$, Andrew R. Deans ${ }^{14}$

I NCSU Insect Museum, Department of Entomology, Box 7613, North Carolina State University, Raleigh, NC 27695-7613 USA 2 American Museum of Natural History, Central Park West at 79th St., New York, NY 10024-5192 USA 3 Species File, Prairie Research Institute, 615 E. Peabody Drive, MC-650, Champaign, IL 61820 USA 4 Department of Entomology, Pennsylvania State University, 501 ASI Building, University Park, PA 16802 USA (starting 1 July 2012) 5 Naturhistoriska Riksmuseet, Department of Entomology, Box 50007, SE-104 05 Stockholm, Sweden 6 Plazi, Zinggstr 16, 3007 Bern, Switzerland 7 Naturhistorisches Museum der Burgergemeinde Bern, Bernastrasse 15, 3005 Bern, Switzerland 8 Australian Centre for Evolutionary Biology \& Biodiversity and School of Earth \& Environmental Sciences, The University of Adelaide, AUSTRALIA 50059 National Evolutionary Synthesis Center, 2024 West Main St., Suite A200, Durham, NC 27705 USA; and Department of Biology, University of North Carolina, Chapel Hill, NC 27599-3280 USA 10 Department of Entomology, One Shields Avenue, University of California at Davis, Davis, California 95616, USA II Department of Entomology, National Museum of Natural History, Smithsonian Institution, P.O. Box 37012, MRC 188, Washington, D.C. 20013-7012, USA 12 Dept. of Entomology, the Natural History Museum, Cromwell Road, London SW7 5BD, UK 13 School of Life Sciences, University of KwaZulu-Natal, Private Bag X01, Scottsville, Pietermaritzburg, 3209 South Africa 14 Department of Evolution, Ecology \& Organismal Biology, The Ohio State University, 1315 Kinnear Road, Columbus, OH 43212, USA I5 Systematic Entomology Laboratory, USDA-ARS, clo NMNH, 10th \& Constitution Ave NW, MRC 168, PO Box 30712, Washington, DC, 20003 USA 16 Canadian National Collection of Insects, Arachnids and Nematodes, AAFC, 960 Carling Ave, Ottawa, Ontario, K1AOC6, Canada 17 Station Linné, Skogsby 161, 386 93 Färjestaden, Sweden 18 Museu de Zoologia da Universidade de Säo Paulo, Av. Nazaré, 481, Ipiranga, 04263-000, São Paulo, SP-Brazil 19 State Museum of Natural History, Entomology, Rosenstein 1, 70191 Stuttgart, Germany 20 Museum fuer Naturkunde, Leibniz-Institut fuer Evolutions- und Biodiversitaetsforschung an der Humboldt-Universitaet zu Berlin, Invalidenstr. 43, D-10115 Berlin, Germany 21 Department of Bioscience, Aarhus University, Ny Munkegade 114, Bldg. 1540, DK-8000, Aarhus C, Denmark 22 Dept.

Copyright Katja C. Seltmann et al. This is an open access article distributed under the terms of the Creative Commons Attribution License 3.0 (CC-BY), which permits unrestricted use, distribution, and reproduction in any medium, provided the original author and source are credited. 
Biodiversity Informatics, Swedish Museum of Natural History, Box 50007, SE-18450 Stockholm, Sweden 23 Department of Biology II, University of Munich (LMU), 82152 Planegg-Martinsried, Germany 24 Department of Entomology, S-227 Agricultural Sciences Bldg North, University of Kentucky, Lexington, Kentucky 40546-0091, USA 25 Natural History Museum of Denmark, University of Copenhagen, Universitetsparken 15, DK-2100 DENMARK 26 Department of Entomology, Texas A\&M University, College Station, TX 77843 USA

Corresponding author: Andrew R. Deans (adeans@gmail.com)

Academic editor: S. Schmidt | Received 25 February 2012 | Accepted 18 April 2012 | Published 31 May 2012

Citation: Seltmann KC, Yoder MJ, Mikó I, Forshage M, Bertone MA, Agosti D, Austin AD, Balhoff JP, Borowiec ML, Brady SG, Broad GR, Brothers DJ, Burks RA, Buffington ML, Campbel HM, Dew KJ, Ernst AF, Fernández-Triana JL, Gates MW, Gibson GAP, Jennings JT, Johnson NF, Karlsson D, Kawada R, Krogmann L, Kula RR, Mullins PL, Ohl M, Rasmussen C, Ronquist F, Schulmeister S, Sharkey MJ, Talamas E, Tucker E, Vilhelmsen L, Ward PS, Wharton RA, Deans AR (2012) A hymenopterists' guide to the Hymenoptera Anatomy Ontology: utility, clarification, and future directions. Journal of Hymenoptera Research 27: 67-88. doi: 10.3897/JHR.27.2961

\section{Abstract}

Hymenoptera exhibit an incredible diversity of phenotypes, the result of -240 million years of evolution and the primary subject of more than 250 years of research. Here we describe the history, development, and utility of the Hymenoptera Anatomy Ontology (HAO) and its associated applications. These resources are designed to facilitate accessible and extensible research on hymenopteran phenotypes. Outreach with the hymenopterist community is of utmost importance to the HAO project, and this paper is a direct response to questions that arose from project workshops. In a concerted attempt to surmount barriers of understanding, especially regarding the format, utility, and development of the HAO, we discuss the roles of homology, "preferred terms", and "structural equivalency". We also outline the use of Universal Resource Identifiers (URIs) and posit that they are a key element necessary for increasing the objectivity and repeatability of science that references hymenopteran anatomy. Pragmatically, we detail a mechanism (the "URI table") by which authors can use URIs to link their published text to the HAO, and we describe an associated tool (the "Analyzer") to derive these tables. These tools, and others, are available through the HAO Portal website (http://portal.hymao.org). We conclude by discussing the future of the HAO with respect to digital publication, cross-taxon ontology alignment, the advent of semantic phenotypes, and community-based curation.

\section{Keywords}

URI, morphology, biodiversity informatics

\section{Background}

Hymenopterists share a common interest, in part, because their research almost invariably requires the study of or reference to hymenopteran anatomy. Which morphological characters are diagnostic or phylogenetically informative? How is behavior manifested morphologically? In what part of the body are certain genes expressed or certain odors detected? Our collective knowledge of this complex data source, however, 
remained decentralized, resulting in a corpus of observations that remained relatively disconnected and dispersed in the literature.

There have been numerous attempts to clarify relevant anatomy, spanning from comprehensive anatomical treatments of character systems across Hymenoptera (e.g., Oeser 1961 for the ovipositor system, Gibson 1985 and Vilhelmsen et al. 2010 for thoracic structures, Vilhelmsen 1996 for preoral cavity in lower Hymenoptera, Schulmeister 2001 for male genitalia), to more focused taxonomic treatments that cover anatomy at a relatively small scale (e.g., Sharkey and Wharton 1997 for Braconidae, Gibson 1997 and Gibson et al. 1998 for Chalcidoidea, Bolton 1994 for Formicidae, and Michener 2000 for Anthophila). There has been proportionally little effort, however, to unify our collective knowledge of hymenopteran anatomy in a way that is both deeply anatomical and broadly taxonomic (but see Richards 1977, Vilhelmsen et al. 2010). Clarification of anatomical terminology is only one benefit of an ontology. As described by Deans et al. (2012), incorporating concepts from an anatomy ontology into our descriptions allows them to be useful for the greater scientific community and creates a corpus of semantic statements about biodiversity. Simply speaking, applying ontology to taxonomy makes our descriptive statements relevant and utilizable by many scientific disciplines, from new prospective students of Hymenoptera to genomic discovery, to many purposes not yet conceptualized.

In 2006, at the $6^{\text {th }}$ International Congress of Hymenopterists meeting in South Africa, a proposal was made to develop a new mechanism for clarifying hymenopteran anatomy: the Hymenoptera Anatomy Ontology (HAO; Deans and Ronquist 2006). Preliminary data and a prototype collaborative ontology editing interface was developed shortly thereafter primarily by ARD and MJY. In 2008, MJY and ARD used these efforts as leverage to secure funding from the United States National Science Foundation's Advances in Biological Informatics program. The applications and approaches described herein, alongside the ontology itself, are products of the ongoing project.

The HAO has since grown into the largest, best illustrated, and most documented multi-species arthropod anatomy ontology and now includes 2055 anatomical concepts and 3622 terms for these concepts, which are extensively annotated (2880 imagebased, sub annotated with 2912 figure annotations; 5686 text-based), with 269 references linking terms to concepts (see Table 1 for definitions of concept/term). These data are derived from existing publications (the HAO database contains $>1000$ ) and ongoing research by $\mathrm{HAO}$ curators and other contributors.

Yoder et al. (2010) described the general development and structure of the HAO and outlined some of the core problems we seek to address through the development and application of an anatomy ontology, including homonymy (same term for different structures), synonymy (different terms for the same structure), and concept drift (application of a term to an increasingly diverse set of structures over time). General ontological issues, specific anatomical challenges and potential applications of the HAO have been discussed at several meetings (2009-2011 Entomological Society of America meetings, $7^{\text {th }}$ International Congress of Hymenopterists) and at workshops 
Table I. Glossary of ontology-related terminology.

\begin{tabular}{|c|c|}
\hline Word & Definition \\
\hline Class & A synonym of concept, in the context of the HAO. \\
\hline Concept & $\begin{array}{l}\text { The idea (primarily the definition and related annotations) that circumscribes our } \\
\text { understanding of an anatomical feature, as denoted by its structural properties. For example, } \\
\text { "The tagma that is located anterior to the thorax" defines the concept commonly referenced } \\
\text { by the term "head". }\end{array}$ \\
\hline $\begin{array}{l}\text { Concept } \\
\text { Drift }\end{array}$ & The application of a term to an increasingly diverse set of structures over time. \\
\hline $\begin{array}{l}\text { Genus- } \\
\text { differentia }\end{array}$ & $\begin{array}{l}\text { A type of definition structured so as to first describe a more inclusive class of concepts (genus) } \\
\text { and then the characteristics differentiating (differentia) it from other children of that concept. } \\
\text { Definitions in this format typically follow the pattern "The } x \text { that is } y . \text {. Biologists will } \\
\text { recognize this as the way Linnaeus structured scientific names. }\end{array}$ \\
\hline Homonym & A term that is used for two or more concepts. \\
\hline Instance & $\begin{array}{l}\text { A real-life exemplar of the concept. For example, the actual physical head of a specific } \\
\text { specimen, perhaps one of many collected by Lubo Masner, is an instance of the HAO class } \\
\text { identified by the URI http://purl.obolibrary.org/obo/HAO_0000397. }\end{array}$ \\
\hline Label & $\begin{array}{l}\text { The HAO has in the past used label and term interchangeably; here we use term to reflect the } \\
\text { broader use of the latter. }\end{array}$ \\
\hline Ontology & $\begin{array}{l}\text { A set of concepts and relationships (properties) pertaining to a particular domain of knowledge } \\
\text { (e.g. hymenopteran anatomy). Ontology is a mechanism of classification. In the case of the } \\
\text { HAO, a goal is to allow a user to classify anatomical structures within a logically consistent } \\
\text { framework. }\end{array}$ \\
\hline $\begin{array}{l}\text { Obsolete } \\
\text { Concept }\end{array}$ & $\begin{array}{l}\text { Once an HAO identifier (e.g. HAO:0000397) is assigned to an anatomical concept, that } \\
\text { concept will not be altered except for misspellings or obvious grammatical errors. If there is a } \\
\text { fundamental problem with a concept definition (and therefore its URI) that concept is made } \\
\text { obsolete but remains resolvable. }\end{array}$ \\
\hline Relationship & $\begin{array}{l}\text { A property (or attribute) shared between two instances (e.g., "mouthparts part_of head"). } \\
\text { Within the HAO, relationships are recorded as linking two concepts, however when the } \\
\text { ontology is applied to real-life examples, the relationships apply to instances of those concepts. } \\
\text { For example, "the eye is not part of the head" (as recorded in the ontology), but rather "my } \\
\text { eye is part of my head". }\end{array}$ \\
\hline $\begin{array}{l}\text { Semantic } \\
\text { Phenotype }\end{array}$ & $\begin{array}{l}\text { Structured annotations that represent observations of the phenome (see Deans et al. 2012). } \\
\text { A semantic phenotype is a descriptive statement that can be used in computation, or some } \\
\text { logical inference. }\end{array}$ \\
\hline Sensu & $\begin{array}{l}\text { A data construct that is a combination of a concept, term, and an associated reference. For } \\
\text { example, Snodgrass (1941) used the term "phallobase" in combination with the concept } \\
\text { http://purl.obolibrary.org/obo/HAO_0000713 ("The anatomical cluster that is composed of } \\
\text { the cupulae, gonostipites and volsellae."). See discussion and definition in Yoder et al. (2010). }\end{array}$ \\
\hline $\begin{array}{l}\text { Structural } \\
\text { Equivalence }\end{array}$ & apnical sameness or structures. \\
\hline Synonyms & $\begin{array}{l}\text { Two or more terms used to reference the same concept. For example, both terms "phallobase" } \\
\text { and "paramere" have been used to reference the concept http://purl.obolibrary.org/obo/ } \\
\text { HAO_0000713. }\end{array}$ \\
\hline Term & $\begin{array}{l}\text { A name (e.g., word, phrase, acronym) representing a concept to a human user. For example, } \\
\text { "head". (compare label) }\end{array}$ \\
\hline URI & $\begin{array}{l}\text { In the context of the HAO, a unique identifier for a concept. HAO URIs are resolvable, i.e., } \\
\text { if used in a web browser additional information will be provided to the person or agent } \\
\text { (other computer) that made the request. For example, http://purl.obolibrary.org/obo/ } \\
\text { HAO_0000713 is the URI for the concept "The anatomical cluster that is composed of the } \\
\text { cupulae, gonostipites and volsellae." }\end{array}$ \\
\hline
\end{tabular}


hosted at North Carolina State University (2010), the Swedish Malaise Trap Project's 3rd Hymenoptera Workshop in Öland, Sweden (2011), and the HAO email list. Based largely on the dialog generated through these interactions we offer here a hymenopterist-centric reintroduction to the $\mathrm{HAO}$, which focuses on describing new tools and on clarifying some frequently asked questions. The topics addressed herein came up in discussion as points of confusion about ontology and were identified as road blocks to fully understand the reasoning behind how the HAO is created and how it would benefit the community beyond a glossary of terms. We attempt to address ontology-related jargon here through our own glossary (Table 1), rather than avoiding the terminology altogether. We also describe how to contribute to the ongoing development of this community resource.

\section{The Hymenoptera Anatomy Ontology Project}

The HAO is more than a simple glossary, as it is formally structured and follows rules of logic (Yoder et al. 2010). The HAO also serves as the core element of a larger "ecosystem" of digital tools designed to facilitate a more rigorous approach to the study of, and reference to, hymenopteran anatomy. In addition to the discussion provided here on format, archiving, and use, readers are encouraged to learn (and share) more about the HAO on the HAO Wiki (http://hymao.org).

\section{Format of the HAO}

The raw, archived representation of an ontology, such as the HAO, is an explicitly formatted and defined text file. Typically these files are in Web Ontology Language (OWL; http://www.w3.org/TR/owl-features) or Open Biomedical Ontology (OBO; http://www.geneontology.org/GO.format.shtmll) format. The OWL family of languages is a general-purpose means of representing knowledge on the Web, and their development is supported by the World Wide Web Consortium (W3C; http://www. w3.org/). OBO specifications are much more narrowly focused and technically simpler, their purpose being to serve the specific needs of biologists who use ontologies. With some exceptions not yet pertinent to the HAO, tools exist to losslessly (i.e., without loss of data/meaning) translate between OWL- and OBO-formatted ontologies, and the HAO can be downloaded in either format (OWL: http://purl.obolibrary.org/ obo/hao.owl or OBO: http://purl.obolibrary.org/obo/hao.obo).

\section{Where to find the HAO}

Hymenopterists will most likely access the HAO through the HAO Portal website (http://portal.hymao.org/; see below), but the ontology itself is accessible through 
several widely used biomedical databases. For example, the HAO is one of many ontologies archived by the Open Biomedical Ontology (OBO) Foundry (http:// www.obofoundry.org). The OBO Foundry supports archiving and development of OWL and $\mathrm{OBO}$ formats as part of an effort to maintain and promote the use of biological ontologies across biological and medical domains. The OBO Foundry also facilitates ontology dissemination and use, and ontologies archived there are automatically made available through other portals such BioPortal and Ontobee (for the HAO versions see http://bioportal.bioontology.org/ontologies/1362 and http://www.ontobee.org/browser/index.php?o=HAO respectively). Our association with the greater biomedical ontology community, especially the National Center for Biomedical Ontology (http://www.bioontology.org/), ensures that the $\mathrm{HAO}$ will be archived for long-term sustainability and distributed for broad use in other domains.

\section{The HAO in the broader community}

Protocols for ontology construction are produced by a large community of ontologists (HAO curators included), some of whom work with anatomy ontologies in practical ways and others who are more theoretical and primarily concerned with the logic inherent in the structure of ontologies. It is important for the HAO, as it is for any anatomy ontology, to be integrated into larger ontology initiatives. As contributors to the larger anatomy ontology community, the collective $\mathrm{HAO}$ is naturally incorporated into a field of research that embraces many biological disciplines and taxa. We benefit from, and contribute to, the efforts of the entire ontology community, including their infrastructure and software advances. By working within larger initiatives we ensure that the $\mathrm{HAO}$ is positioned to take advantage of ongoing initiatives (e.g. integration with the genomics community).

\section{The HAO "ecosystem"}

The principal product of the HAO Project is the ontology itself, but the process of development has also resulted in multiple applications, built upon the ontology, that facilitate Hymenoptera research. HAO developers are responsible for creating human-usable Web interfaces and applications that make the text-file ontology into tools for the biological community, and to encourage an ontology-based approach to morphology. The primary application is the Hymenoptera Anatomy Ontology Portal (formerly the "Hymenoptera Glossary"). The HAO Portal displays data contained within the Hymenoptera Anatomy Ontology, as well as other information useful for Hymenoptera research including annotated images, alternative definitions, comments from users, ontology visualization tools, an extensive bibliography, and the "analyzer" tool (described below). 


\section{Unique identifiers for anatomical concepts}

Most hymenopterists are familiar with the process of submitting sequences to GenBank and receiving unique identifiers (i.e., GenBank accession numbers) that serve as published references to their data. The HAO serves a similar function for anatomy by providing unique identifiers for anatomical concepts. The identifiers used by the HAO are Uniform Resource Identifiers (URIs) that consist of a Persistent Uniform Resource Locator (PURL: http://purl.obolibrary.org/obo/) plus an HAO identifier in OBO format (e.g. HAO:0000397). The latter is a combination of the namespace used in the OBO Foundry ("HAO") and a unique seven-digit number (e.g. "0000397"). The ":" in the identifier is replaced with an " " in the URL form. Any given URI points to only a single concept within the HAO (e.g. http://purl.obolibrary.org/obo/ HAO_0000397 = "The tagma that is located anterior to the thorax" = head).

\section{The virtue of URIs}

The concept of the URI is important because it allows the HAO to be utilized in multiple contexts. Within Web browsers (e.g. Firefox, Google Chrome, Internet Explorer) many URIs (including the HAO's) may be used in the same manner as URLs (Uniform Resource Locators) for webpages. URIs, in combination with Web-server configurations, allow for different responses, based on who is making the request. For example, a request from a Web browser-a person clicking on a link in a journal article or website-would return content that a human can interpret (e.g., a webpage), whereas requests from computational sources would receive responses that the application can understand (leaving out the human-readable components). This core functionality is at the heart of the long-term use and application of HAO URIs within publications, websites, applications and analyses. The challenge then is to facilitate the production, use, and publication of URIs (see below).

\section{Referencing the HAO in publications}

\section{The URI Table}

Materials and methods sections within papers that reference hymenopteran anatomy frequently point to concepts published elsewhere (e.g., "Morphological terminology largely follows Mikó et al. 2007” in Talamas et al. 2011). New or revised anatomical concepts are typically paragraphical, with terms highlighted and a definition or discussion following (e.g. Yoder 2004). Here we introduce an alternative, table-based format that facilitates reference to existing anatomical concepts within the HAO. Examples exist in Johnson and Musetti 2011, Mikó et al. 2012, and Talamas et al. 2011. In its simplest format the table only requires two columns: a term and a URI. The format, 
including its required elements and additional optional extensions (up to six total columns), is exemplified in Table 2. An explanation of the columns follows:

1. Term: The literal text (string of letters) used in the paper for the anatomical concept. It is important to reference anatomical terms used within the table exactly throughout a paper, to eliminate ambiguity. Simplification of terms within a document, without including these simplifications in the table, reduces the effectiveness of a table intended to clarify terminology. For example, if "abdominal tergum 3 " is in the table, the structure should be fully referenced throughout the document as "abdominal tergum 3" and not simplified to "the tergum" anywhere.

2. URI: The unique, resolvable, identifier for the anatomical concept. HAO curators create these identifiers, which can be found by accessing the HAO Portal.

3. Definition: A verbatim replication of the definition in the HAO, which are written as genus-differentia (see Smith 2005).

4. Definition source: A citation from which the concept/definition was derived; within the $\mathrm{HAO}$

5. HAO preferred term: The present preferred term for the concept denoted by the URI in the HAO (see below for explanation). This term is provided only when it does not match the term the author uses in his or her publication. This option provides authors the freedom to reference anatomy in any manner he or she sees fit.

6. Comment: Comments may pertain to any or all of the columns for a given concept. For example they can be used to clarify subtleties or provide taxon-specific discussion.

Table 2. An example URI table. Starred $\left({ }^{*}\right)$ columns are required. See discussion for details.

\begin{tabular}{|c|c|c|c|c|c|}
\hline Term* & URI* & Definition & $\begin{array}{l}\text { Definition } \\
\text { Source }\end{array}$ & \begin{tabular}{|c|} 
HAO \\
Preferred \\
term
\end{tabular} & Comment \\
\hline head & $\begin{array}{c}\text { http://purl. } \\
\text { obolibrary.org/obo/ } \\
\text { HAO_0000397 }\end{array}$ & $\begin{array}{l}\text { The tagma } \\
\text { that is located } \\
\text { anterior to the } \\
\text { thorax. }\end{array}$ & $\begin{array}{c}\text { Snodgrass, R. E. } \\
1935 .\end{array}$ & & \\
\hline paramere & $\begin{array}{c}\text { http://purl. } \\
\text { obolibrary.org/obo/ } \\
\text { HAO_0000395 }\end{array}$ & $\begin{array}{l}\text { The sclerite that } \\
\text { is connected } \\
\text { distally to the } \\
\text { gonostipes. }\end{array}$ & $\begin{array}{c}\text { Crampton, G. } \\
\text { C. } 1919 .\end{array}$ & harpe & \\
\hline propodeum & $\begin{array}{c}\text { http://purl. } \\
\text { obolibrary.org/obo/ } \\
\text { HAO_0001249 }\end{array}$ & $\begin{array}{l}\text { The area that } \\
\text { is located } \\
\text { posterior to the } \\
\text { metapleural } \\
\text { carina. }\end{array}$ & $\begin{array}{l}\text { Mikó, I., } \\
\text { L. Vilhelmsen, } \\
\text { N. F. Johnson, } \\
\text { L. Masner, and } \\
\text { Z. Pénzes. 2007 }\end{array}$ & & $\begin{array}{l}\text { The propodeum here } \\
\text { is also part_of the } \\
\text { metapectal-propodeal } \\
\text { complex- this further } \\
\text { bounds the somewhat } \\
\text { generalized defintion. }\end{array}$ \\
\hline
\end{tabular}




\section{Creating a URI table}

URI tables are created (Fig. 1) prior to submission of a manuscript, usually in close conjunction with an $\mathrm{HAO}$ curator, though they can also be built independently. The goal of building a URI table is to find or help create a URI that represents the anatomical structures referenced in a paper (though a URI table that only references a subset is still useful; see Wharton et al. 2010). The highest priority candidates for a URI table are those structures that have historically been poorly defined, new concepts, or those that have synonymous and/or homonymous terms. Authors will typically reference more than one anatomical character or feature (= "pool of anatomical concepts" in Fig. 1:1) in a manuscript. This pool typically comes from the description and diagnosis sections of a manuscript.

The first step in building a URI table involves discovering available URIs (Fig 1:2), using tools available at the HAO Portal site: the simple Portal search tool, the "analyzer tool" (described in detail below), or by requesting help from the HAO e-mail list. If URIs are found they can be added to the table (Fig 1:3) after the user is satisfied with the HAO concept, citation, and labels provided. If a match is not found, or the author is not satisfied with a present concept, an HAO curator will work with the author to create a new URI (Fig 1:4). HAO curators can be contacted through the feedback form online (links from http://portal.hymao.org), through email directly to an HAO curator, or through contacting the broader HAO community on the listserv (http://purl.oclc.org/NET/hymontology/listserv). Authors are cautioned when reviewing concepts to watch for homonymy and synonymy, and not to assume that if a term match is returned that the definition will also match the authors notion of its meaning in the manuscript (Fig 1:5). In other words, authors should carefully review term definitions before adding them to the URI table. Tables can be included in Materials and Methods sections or as appendices, preferably as part of the manuscript rather than supplementary documents (Fig 1:6). While URI tables can be developed independently it is recommended that you pass along your table to the HAO curators to review prior to submission for publication. The entire process is iterative (Fig 1:7) and may be repeated multiple times as a manuscript develops.

\section{Using the "analyzer" tool}

The goal of the analyzer tool (Fig 1:2) is to facilitate the adoption of URIs in publications and digital applications. The mechanism created to accomplish this is a simple interface (Fig. 2) for submitting an author's anatomy-related text. The provided text is broken down into individual and groups of words, and these are compared against the terms in the HAO for matches by an internal algorithm. Terms are matched letter-forletter, i.e., "forewing" will not match "fore wing". No "fuzzy" matching attempts are made, i.e., the software does not try to predict what anatomical concepts the author is referring to. Matching terms are summarized by the anatomical concept to which they are attached (Fig. 2D). URIs for each matching concept are also provided. To use the analyzer follow these steps and see the online help (Fig. 2A, "What is this?"): 


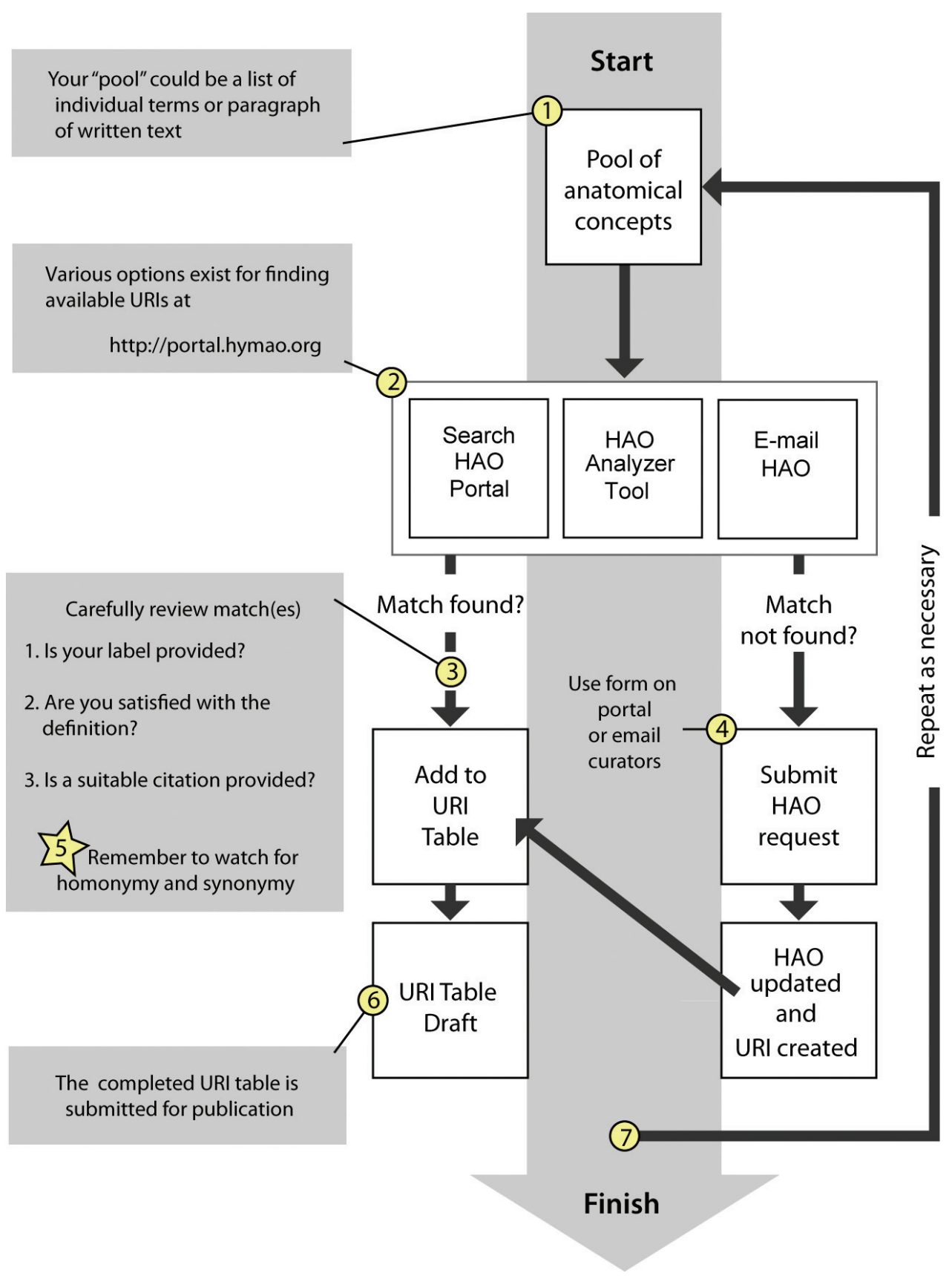

Figure I. Workflow for drafting a URI table.

1. Provide your text: Copy and paste your text, or type directly into the text box (Fig. $2 \mathrm{~B})$. Try to limit the text in the box to that which is pertinent to the underlying ontology being analyzed (e.g., do not include specimen examined sections from 
taxonomic descriptions, but rather the description alone). You can paste sentences, entire paragraphs from a manuscript description or diagnosis, comma-delimited lists, or any combination thereof. Typically, this would include a description and diagnosis from the manuscript.

2. Prove you are human: To discourage spammers and other troublemakers the analyzer form includes a "CAPTCHA" (http://www.captcha.net/) (Fig. 2C). Type the two words shown into the text box. If you need help click the small "?" on the form. Each time you click the submit button you will have to re-complete the CAPTCHA, even after you have successfully filled one out the first time.

3. Submit: Click Submit and wait (you will see a spinner turning). You might have to wait for a few seconds depending on the amount of text submitted.

4. Examine results: Results are displayed in a table (Fig. 2D) below the form. Be aware that if a term is not in the HAO it will not appear in the results list. Use the "Match Map" from the Detailed Breakdown Report (see Fig. 3) to check if all of your terms are included. References (Fig. 2, "References") represent citations wherein the term was used in conjunction with the definition/concept as interpreted by an HAO curator. Carefully reviewing each conceptual match is critical; do not assume that the result is using the term you have provided in the manner you mean. Feedback based on missing terms, problems with definitions, etc. can be provided as outlined in Fig. 1 (and see Curators and Curation below). An additional breakdown of the results (Fig. 1F; Fig. 2) provides more details.

5. Download results: Download the result by clicking the "Download" button (Fig. $1 \mathrm{E})$. The downloaded file is a tab-delimited file that can be opened directly in a spreadsheet program (e.g., Microsoft Excel) or a text editor.

6. Edit the results: If multiple concepts (definitions) are available for a given term choose the one that matches your concept. If you need help determining which concept to select use a feedback mechanism (Fig. 1). Delete the other rows. Repeat for all the terms in the table.

7. See more: For additional details click on "Detailed breakdown" (Fig. 1F; Fig. 3). This report can be used to visually inspect for misformatted terms, alternate spellings or usages of a term, or potentially new additions to the HAO. The report includes:

a. Analyzed Text: Use this to confirm that your text was submitted correctly. The analyzer may truncate your text if it is too long, or if other problems are encountered.

b. Match Map: The words that were matched and returned are highlighted in the context of the text here. Green highlights indicate a 1:1 mapping (i.e., there is only one concept present for the given term). Pink highlights indicate that there are multiple concepts for a given term.

c. Matched Terms: A simple comma separated list of the terms that were found.

d. Matched Classes: A list of the concepts that were matched.

e. Homonymous Terms: Matched terms that are homonyms.

f. Synonymous Terms: Matched terms that are synonyms. 


\section{Analyze}

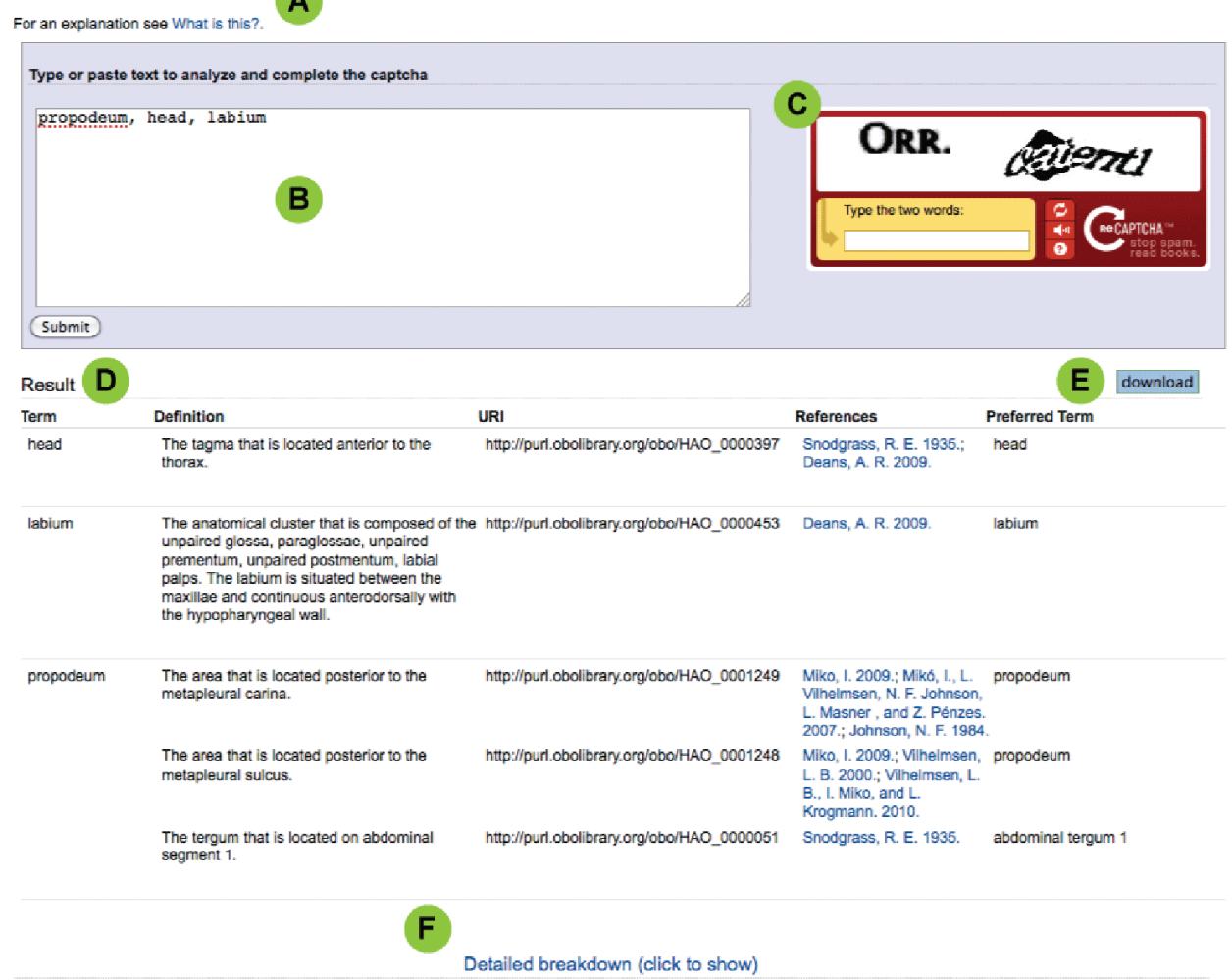

Figure 2. Using and interpreting results from the analyzer. See Using the "analyzer" tool for explanation. A Help link B Input field C CAPTCHA test D Result table E Download link; and F Detailed breakdown link.

\section{Recurrent Issues}

The HAO project is exemplary in its close connection to domain experts (hymenopterists), and its success depends on broad community support. During its short life, the potential utility of the HAO has already begun to emerge (e.g., Mikó et al. 2012, Johnson and Musetti 2011, Kawada 2011, Talamas et al. 2011, Mikó et al. 2010, Vilhelmsen et al. 2010, Mikó and Deans 2009, Deans and Kawada 2008, Yoder 2007). Here we attempt to clarify recurrent issues, with the goal of facilitating continued adoption of the HAO. The topics listed here result from input from many of the authors of this paper. These interactions, facilitated by the HAO email listserv, HAO Portal feedback form, workshops, and personal interactions, guided the structure of this manuscript, as well as the development of the "analyzer" tool. Integration and reference to the HAO should not be interpreted as "additional work" but rather as "due diligence". Simply put, when an author references anatomical concepts s/he needs to ensure that those references will be consistently interpreted. Utilizing the $\mathrm{HAO}$ can help ensure this goal is realized. 
Detailed breakdown (click to show)

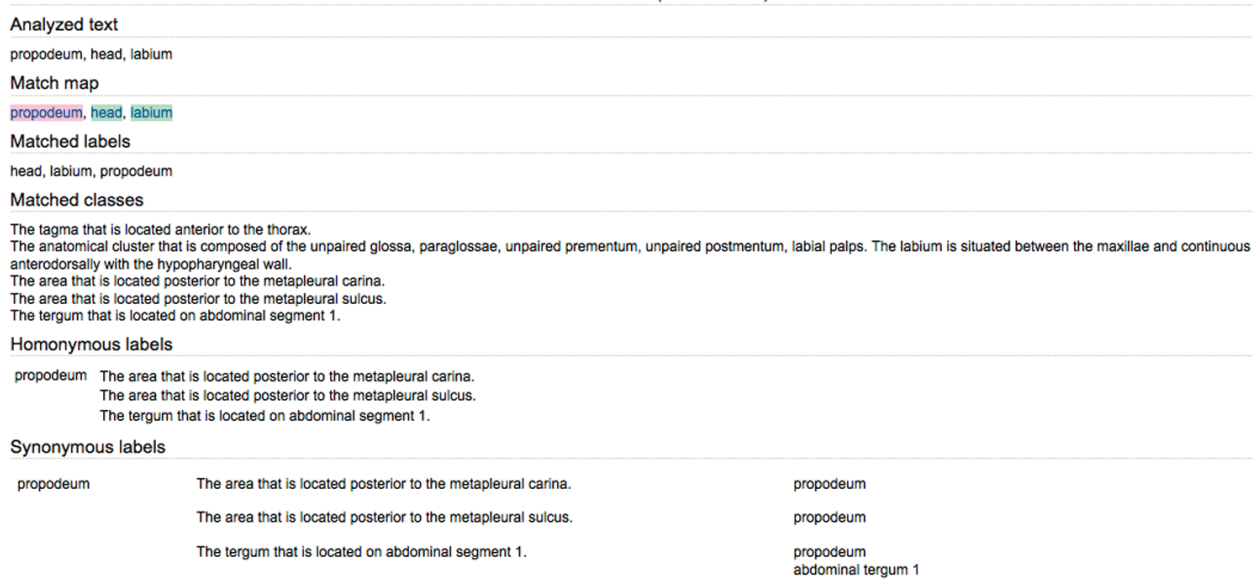

Figure 3. The detailed breakdown report from an analyzer report. See Using the "analyzer" tool for explanation.

\section{Homology and the HAO}

Understanding the criteria for defining HAO concepts is important for understanding the decision-making process for constructing the $\mathrm{HAO}$ and how it can be useful as a tool for referencing and aligning anatomical concepts. Fundamentally, the $\mathrm{HAO}$ project rests on recognizing different instances of a topographically-defined concept as "the same" (e.g., the fore wing of taxon A is the same structure as the fore wing of taxon B) (topographical homology of Jardine 1969 and Rieppel 1980; topographical correspondence of Rieppel 1988 and De Pinna 1991; topographical identity of Brower and Schawaroch 1996; homology of Owen 1843 and Remane 1952). The HAO employs the principle of "structural equivalence" to discuss topographical sameness. In biology, however, homology is often more explicit, referring to a more profound "sameness", because it expresses a theory about structures sharing a common evolutionary origin even if they appear structurally dissimilar (homology of Patterson 1982, Nixon and Carpenter 2011, secondary homology of de Pinna 1991, Brower and Schawaroch 1996, historical homology of Wagner 1989, taxic homology of Haszprunar 1992, Scotland 2000, Wiley and Lieberman 2011, homogeny of Lankester 1870, "Darwinian homology"). Homology in this evolutionary context is often dynamic, and may be controversial or involve conflicting hypotheses or quickly changing views. The dynamic nature of homology hypotheses conflicts with the HAO's goal of unambiguous circumscription of anatomical concepts, and, as such, overt reference to homology hypotheses are avoided in constructing $\mathrm{HAO}$ definitions. 
The HAO provides structure-based anatomical concepts, from which homology hypotheses can be developed and subsequently tested. This strategy derives from the premise that statements of homology, in the absence of phylogenetic tests, should be avoided. This is not to say that the construction of the HAO is not influenced by (and can influence) phylogeny-based homology hypotheses. New concepts can be derived as necessary, but these concepts will be rooted in what can be observed, not in what is hypothesized. One of the HAO's greatest strengths is that it forces the careful introspection necessary for generating strong primary homology hypotheses by forcing users to reconcile a localized understanding (e.g. an author's unwritten understanding of the anatomical nuances) with a broader conceptual framework. Many HAO concepts are sufficient for the basis of evolutionary homology hypotheses; however this is not always the case.

One example of how a structurally equivalent concept might not be homologous pertains to processes (http://purl.obolibrary.org/obo/HAO_0000822). Many distantly related hymenopterans have similarly located (i.e. structurally equivalent) pronotal spines. These spines would correspond to the same concept in the HAO, i.e., they are analogous but are not necessarily homologous.

\section{Classifying anatomical structures using structural equivalency}

Structural equivalency allows clustering of anatomical structures described in the literature, based on clear, repeatable criteria-i.e. the criteria are observable, not hypothesized. Because of the structural equivalency criterion, the match between ontology concept and author concept of a term does not need to be exact but rather only structurally equivalent. HAO definitions, then, do not include all of the nuances of a concept; only the author can articulate those. When an author's concept of a term (based on observations pertaining to its structure) differs from that of the definition in the $\mathrm{HAO}$ (including its properties) a new concept should be created.

If an author intends to state that a particular anatomical concept was observed in some instance of a hymenopteran then that author must ensure that they observed all of the components of the anatomical concept provided in the HAO for that concept. For example, in many HAO concepts points of muscle attachment are used (an important line of evidence for topographical definitions). The author must have observed these muscles and their points of attachment to state that the class in question was present in their hymenopteran instance. Careful attention must also be made to the relationships a particular anatomical concept has to other concepts; these relationships must also be observed by the author.

There is, however, one circumstance in which anatomical concepts in the HAO might be "loosely referenced" (e.g., referencing an anatomical concept that includes, in part, reference to muscle arrangements when said muscle arrangements have not been observed). This is during the formulation or discussion of hypotheses, often pertaining to character evolution. For example, a paleontologist studying two fossils may wish to 
discuss them in the context of anatomical concepts in the HAO: "I hypothesize that fossil one bears anatomical concept HAO 1234 except for feature A (which I cannot observe based on preservation), and fossil two bears anatomical concept HAO 1234 except for feature B (which I believe is absent, but I would like to see more specimens)". In this hypothetical scenario minting two new HAO URIs (i.e., HAO 1234 minus feature A, HAO 1234 minus feature B), gains us little (and may be positively misleading based on the uncertainty of the observer), in the absence of additional observations. The referencing to HAO 1234, however, provides an anchor to a discussion of the evolution of an anatomical concept, something potentially of use to future researchers.

\section{Separation of terms and concepts}

Terms and concepts are treated separately, and only concepts are given unique identifiers. Terms in the HAO are considered to be simply strings of letters that are used to reference concepts, associated together because they are structurally equivalent anatomical structures. Concepts encapsulate meaningful anatomical observations. Different observers looking at the same anatomical structure can have the same conceptualization of it, where it is located on the body, and the circumscription of area around it. Yet even with this similarity, they may still use different terms for the observed anatomical structure (synonymy). Different observers may use the same term yet have different understandings of when and how that term is applicable (the "sensu" construct of Yoder et al. 2010), or the same term may be used for different anatomical concepts (homonymy). The separation of concept from term is important for understanding how the HAO is useful for clarification of terminology.

\section{Preferred terms}

Because there are no international rules of anatomical nomenclature, as there are for zoological nomenclature, anyone can use any terms they want to refer to an anatomical concept of interest. The HAO stands as a robust mechanism (through URIs) to clarify diverse concepts. Given the dual legacies of published literature and training histories, we envision a community where personalized lexicons continue to be refined and accommodated. Many hymenopterists, however, including a group at the 7 th International Congress of Hymenopterists (Köszeg, Hungary; June 2010), have called for the HAO Project to serve as an instrument for deciding upon preferred terms for concepts. Preferred terms facilitate accurate communication by simplifying concordance of terms with concepts, and they are required by potential HAO users in biomedicine.

Terms in a manuscript are strings of letters that may or not have meaning to a reader depending on his or her knowledge. A reader experienced enough with the terminology typically used to describe a certain taxon will not necessarily need to check the attached URI table to understand the meaning of a term. For a non-expert reader, unfamiliar 
with terms in a manuscript, preferred terms may be meaningless (or even misleading). However, preferred terms are very important for experts, who use "short" terms to encompass a hidden mountain of unexpressed background information. Preferred terms also are of use to taxonomists in the early stages of developing a vocabulary for their taxa, as the set is much smaller than the total number of possibilities. If an aspiring taxonomist can navigate directly to this set they potentially become more efficient in its use and less confused by the historical baggage carried by a larger set of overlapping terms.

The HAO now has an accessible listserv (http://purl.oclc.org/net/hymontology/ listserv) for preferred term voting. Preferred term choices are emailed to the list once or twice a week and the votes are collected with the term obtaining the most votes moved into the "preferred" position on the HAO Portal webpages. All other terms are then considered synonyms. The "term of the week" is selected based on the frequency of term usage found in past issues of the Journal of Hymenoptera Research. As of this writing, listserv members have voted on about 60 preferred terms, all of which may be revisited by calling for a new discussion on the listserv.

\section{Why not simply include definitions in one's paper?}

Ontologies have structure, and concept definitions therefore inherit the definitions of all the concepts contained within the definition and the concepts it is related to. Authors themselves cannot easily explicitly define all the terms used in a publication, as their definitions will inevitably include terms that must also be defined. To precisely define one concept requires an entire ontology and one that is internally consistent (i.e. only uses terms that are also defined in the ontology). In addition, the HAO project provides a wealth of additional information. By linking to concepts in the HAO authors not only explicitly define terms in their publications, but the reader also gets other information about the concept, such as which major publications used the concept, its synonyms, homonyms, annotated images, and additional comments from the HAO curators.

\section{Persistence}

Our ideas of concepts may change over time, but the original concepts will never disappear in HAO. Once an HAO identifier (e.g. HAO:0000397) is assigned to an anatomical concept, that concept will not be altered except for misspellings or obvious grammatical errors. If there is a fundamental problem with a concept definition (and therefore its URI) that concept is made obsolete. Links to the obsolete concept persist, however, so that published URIs will always resolve, regardless of their standing in the current HAO. Obsolete classes in the HAO require statements as to why they were made obsolete and links to the newly derived concepts where pertinent. The obsoletion mechanism, in conjunction with a pluralistic approach to providing concepts (i.e., it is not a problem to create subtly different concepts), are strategies to minimize concept drift. 


\section{Web-browsers}

Some functionality of the HAO Portal currently does not work for some Web browsers. The HAO Project strongly recommends using Firefox (http://firefox.org) to view the HAO Portal pages in order to assure the proper rendering of annotated images. The HAO Portal utilizes cutting-edge functionality that some browsers (e.g., Internet Explorer, IE) presently do not support. However, if a person accesses a webpage in IE a warning message will appear directing the user to open the page in Firefox. Because free, standards-compliant browsers are available we do not see this requirement as a critical limitation. We anticipate that advances in browser capabilities related to the advent of the HTML5 standard will ensure that the HAO Portal will shortly truly become cross-browser compatible.

\section{The Future of the HAO}

The HAO is a resource based on a foundation of explicitly defined anatomical concepts and a straightforward mechanism for dereferencing these concepts (URIs). This resource is intended for any and all users who reference Hymenoptera anatomy. The $\mathrm{HAO}$, like other biological ontology efforts, is rapidly evolving, both in its underlying data and its application. Its adoption depends first and foremost on the support of the hymenopterist community. The HAO is poised to succeed thanks, in part, to technological advances in publication, ontology alignment, and the burgeoning potential of semantic phenotypes (Deans et al. 2012).

\section{HAO in Publications}

Publications that precisely illustrate morphology routinely become the most cited and useful papers for future hymenopterists. The Journal of Hymenoptera Research $(J H R)$ serves many experts and is well positioned to benefit from the rapidly growing publishing tool kit. The open-access model adopted by the JHR embraces the Web, pushing publications out of the traditional print-only format. Additionally, the JHR is published by Pensoft Press, which uses the TaxPub XML schema (Catapano 2010) to mark-up manuscripts and subsequently transform them through semantic enhancements (Penev et al. 2010). There is amazing potential for hymenopterists created by the connection of these elements and those introduced by the HAO Project. As demonstrated multiple times from output of the vSysLab workbench (http://vsyslab.osu. edu; Johnson 2010), TaxPub marked-up text for descriptions can be generated directly from a database, without additional formatting headaches. The resulting XML already explicitly identifies which sections of a publication are the descriptions of new taxa. Names are automatically sent to ZooBank (the International Commission on Zoological Nomenclature name-registration service), sequence data to the National Center for 
Biotechnology Information (GenBank), and information is aggregated from markedup publications by the Global Biodiversity Information Facility (GBIF) and the Encyclopedia of Life (EOL). HAO and TaxPub developers are working to integrate the URI links into the schema; thus not only will the portions of the text be identified as the description but also the terminology used in that block of text can be explicitly defined.

\section{Alignment}

As ontologies continue to be adopted beyond the model-organism community they will be increasingly cross-referenced. Precisely defined concepts for hymenopteran anatomy, for example, can be connected to anatomical concepts in other domains that also have an ontology (e.g. Diptera). These alignments have the potential to facilitate novel evolutionary developmental biology hypotheses and hypotheses of homology. For example, while aligning the muscles in the FlyBase anatomy ontology (Drosophila; http://flybase.org/) with those concepts in the HAO, HAO developers noticed that the muscle patterns of the hymenopteran meso- and metathorax share similarities with the metathorax of different Drosophila Ultrabithorax (Ubx) mutants (see Rivlin et al. 2001). This approach is extensible to numerous other genotype-phenotype comparisons, and knowledge derived from Drosophila (or other model-organism) mutant phenotypes stands to shed light on Hymenoptera evolution. This avenue of discovery, building on the well-founded work in other model-organism communities, is made possible with well-aligned anatomy ontologies.

The process of alignment and integration across ontologies is itself an intellectually challenging endeavor. Work facilitated by the Phenotype RCN (http://www.phenotypercn.org), which includes several HAO developers, is supporting the development of new arthropod-specific ontologies (e.g. one each for Coleoptera, Neuroptera, and Arthropoda). Work of this nature and scope is novel and will undoubtedly lead to big discoveries and new interpretations.

\section{Semantic phenotypes}

Deans et al. (2012) recently expressed the opinion that taxonomic diagnoses and descriptions (and perhaps other prosaic descriptions of phenotypes) should be transformed by new approaches to derive semantic phenotype (Table 1) statements. Their premise was that taxonomic descriptions (the actual descriptive text found in a taxon description or diagnosis text) are essentially inaccessible in their current form. If these data are recorded in a semantic format, however, referencing the HAO and other phenotype-relevant ontologies, they would be accessible to researchers in other domains in what is clearly an increasingly digital world. Pragmatically speaking, we are not quite prepared to complete this transformation, but hymenopterists are well positioned to 
be on the cutting edge of this endeavor thanks, in part, to the existence of a robust, domain-specific anatomy ontology.

\section{Curators and curation}

No formal organization currently governs the HAO, but the International Society of Hymenopterists has extended their support in making sure its future is secure. An ontology working group was formed (see ISH newsletter, March 2006), and the Society has offered to host HAO resources through their website should that service be desired by the community. The de facto curators (IM, MJY, ARD, KCS, MAB) strongly support community input and governance and will work with stakeholders to develop mechanisms for recruiting other curators and for facilitating decisions about ontology development (which must follow the recommendations of the broader ontology community, mainly the National Center for Biomedical Ontology and the OBO Foundry principles, to ensure robustness and broad use).

\section{Conclusion}

In addition to increasing the repeatability of our research, references to well-defined and illustrated anatomical concepts will open up their interpretation and use to a much broader array of users than just highly specialized scientists. Biodiversity, host-parasite biology, collections digitization, genomics, ecology, evolutionary developmental biology (evo-devo), invasive species evaluation, agro-ecosystem management and biological control (to name just a few) as well as those aspects of society impacted by these all rely on the correct interpretation of anatomical structures (e.g., Mikó et al. 2012). The core mechanism by which one can reference the HAO, URIs, is implementable now via simple URI tables. Much more needs to be done to transparently and seamlessly integrate the HAO into the scientists' workflow for maximum benefit of scientists and the users of their science, but the basic mechanism, URIs, will be central to these emerging methods. As we move forward into an exciting period of exploding new technologies and exploration we ask that the hymenopterist community continue to participate in the growth, refinement, and evolution of the HAO.

\section{Acknowledgments}

This work was made possible by the U.S. National Science Foundation, grants DBI0850223 and DEB-0842289, by a grant from the Encyclopedia of Life's Biodiversity Synthesis Center (BioSynC), and by the Phenotype Research Coordination Network (NSF DEB-0956049). USDA is an equal opportunity provider and employer. 


\section{References}

Agosti D, Egloff W (2009) Taxonomic information exchange and copyright: the Plazi approach. BMC Research Notes 2153. doi: 10.1186/1756-0500-2-53

Bolton B (1994) Identification Guide to the Ant Genera of the World. Harvard University Press, Cambridge, Massachusetts.

Catapano T (2010) TaxPub: An extension of the NLM/NCBI Journal Publishing DTD for Taxonomic Descriptions. Proceedings of the Journal Article Tag Suite Conference 2010 [Internet]. Bethesda, MD: National Center for Biotechnology Information (US).

Deans AR, Yoder MJ, Balhoff J (2012) Time to change how we describe biodiversity. Trends in Ecology and Evolution 27 (2): 78-84 doi: 10.1016/j.tree.2011.11.007

Deans AR, Ronquist F (2006) Ontologizing morphological terms for Hymenoptera - implementing and benefiting from a controlled vocabulary. 6th International Conference of Hymenopterists, Sun City South Africa. January 2006.

Deans AR, Kawada R (2008) Alobevania, a new genus of neotropical ensign wasps (Hymenoptera: Evaniidae), with three new species: integrating taxonomy with the World Wide Web. Zootaxa 1787: 28-44.

Gibson GAP, Read JD, Fairchild R (1998) Chalcid wasps (Chalcidoidea): illustrated glossary of positional and morphological terms. http://www.canacoll.org/Hym/Staff/Gibson/apss/ chglintr.htm

Gibson GAP (1985) Some pro- and mesothoracic structures important for phylogenetic analysis of Hymenoptera, with a review of the terms used for the structures. Canadian Entomologist 117: 1395-1443.

Gibson GAP (1997) Morphology and terminology. In: Gibson GAP, Huber JT, Woolle JB (Eds) Annotated keys to the genera of Neartctic Chalcidoidea (Hymenoptera). NRC Research Press, Ottawa: 16-44.

Goulet H, Huber JT (1993) Hymenoptera of the World: An Identification Guide to Families. Research Branch, Agriculture Canada Publication 1894/E. Ottawa, Ontario, 1-668.

Haszprunar G (1992) The types of homology and their significance for evolutionary biology and phylogenetics. Journal of Evolutionary Biology 5: 13-24.

Jardine N (1969) The observational and theoretical components of homology: a study on the morphology of the dermal skull-roofs of rhipidistian fishes. Biological Journal of the Linnean Society 1: 327-361.

Johnson N (2010) Future taxonomy today: new tools applied to accelerate the taxonomic process. In Polaszek (Ed) Systema Naturae 250: The Linnaean ark. CRC Press Taylor \& Francis Group, London: 137-147.

Johnson NF, Musetti L (2011) Redescription and revision of the Neotropical genus Pseudoheptascelio Szabó (Hymenoptera, Platygastridae, Scelioninae), parasitoids of eggs of shorthorned grasshoppers (Orthoptera, Acrididae). Zookeys 136: 93-112.

Kawada R (2011) Pictorial key for females of Decevania Huben (Hymenoptera, Evaniidae) and description of a new species. ZooKeys 116: 59-84. doi: 10.3897/ZooKeys.116.1473 
Lankester ER (1870) On the use of the term homology in modern zoology, and the distinction between homogenetic and homoplastic agreements. Annals and Magazine of Natural History 4: 34-43.

Michener CD (2000) The Bees of the World. Johns Hopkins University Press (Baltimore, MD): 1-913.

Mikó I, Vilhelmsen L, Johnson NF, Masner L, Pénzes Z (2007) Morphology of Scelionidae (Hymenoptera: Platygastroidea): head and mesosoma. Zootaxa 1571:1-78.

Mikó I, Deans AR (2009) Masner, a new genus of Ceraphronidae (Hymenoptera: Ceraphronoidea) described using controlled vocabularies. ZooKeys 20: 127-153 doi: 10.3897/zookeys.20.119

Mikó I, Friedrich F, Yoder MJ, Hines HM, Deitz LL, Bertone MA, Seltmann KC, Wallace MS, Deans AR (2012) On dorsal prothoracic appendages in treehoppers (Hemiptera: Membracidae) and the nature of morphological evidence. PLoS ONE 7: e30137 doi: 10.1371/ journal.pone.0030137

Mikó I, Masner L, Deans AR (2010) World revision of Xenomerus Walker (Hymenoptera: Platygastridae). Zootaxa 2708: 1-73.

Nixon KC, Carpenter JM (2011) On homology. Cladistics 27: 1-10. doi: 10.1111/j.10960031.2011.00371.x

Oeser R (1961) Vergleichend-morphologische Untersuchungen über den Ovipositor der Hymenopteren. Mitteilungen aus dem Zoologischen Museum in Berlin 37: 3-119.

Owen R (1843) Lectures on the Comparative Anatomy and Physiology of the Invertebrate Animals. Longman, Brown, Green and Longmans (London).

Patterson C (1982) Morphological characters and homology. In Joysey KA, Friday AE (Eds) Problems of Phylogenetic Reconstruction. Academic Press, London \& New York: 21-74.

Penev L, Agosti D, Georgiev T, Catapano T, Miller J, Blagoderov V, Roberts D, Smith V, Brake I, Ryrcroft S, Scott B, Johnson N, Morris R, Sautter G, Chavan V, Robertson T, Remsen D, Stoev P, Parr C, Knapp S, Kress W, Thompson C, Erwin T (2010) Semantic tagging of and semantic enhancements to systematics papers: ZooKeys working examples. ZooKeys 50: 1-16. doi: 10.3897/zookeys.50.538

de Pinna MGG (1991) Concepts and tests of homology in the cladistic paradigm. Cladistics 7: 367-394.

Remane A (1952) Die Grundlagen des natürlichen Systems, der vergleichenden Anatomie und der Phylogenetik. Geest und Portig, Leipzig.

Richards OW (1977) Hymenoptera. Introduction and key to families. Handbooks for the Identification of British Insects 6 (1) (2nd ed.): 1-100.

Rieppel O (1980) Homology, a deductive concept? Zeitschrift für Zoologische Systematik und Evolutionsforschung 18: 315-319.

Rieppel O (1988) Fundamentals of Comparative Biolog. Birkhauser, Basel.

Rivlin PK, Gong A, Schneiderman AM, Booker R (2001) The role of Ultrabithorax in the patterning of adult thoracic muscles in Drosophila melanogaster. Development Genes and Evolution 211: 55-66. doi: 10.1007/s004270000126 
Schulmeister S (2001) Functional morphology of the male genitalia and copulation in lower Hymenoptera, with special emphasis on the Tenthredinoidea s. str. (Insecta, Hymenoptera, 'Symphyta'). Acta Zoologica 82: 331-349.

Scotland RW (2000) Taxic homology and three-taxon statement analysis. Systematic Biology 49: 480-500.

Sharkey MJ, Wharton RA (1997) Morphology and terminology. In: Wharton RA, Marsh PM, Sharkey MJ (Eds) Manual of the New World genera of Braconidae (Hymenoptera). Special Publication of the International Society of Hymenopterists. Vol. 1: 19-38.

Smith EL (1970) Evolutionary morphology of external insect genitalia 2 Hymenoptera. Annals of the Entomological Society of America 63:1-27.

Smith B (2005) The logic of biological classification and the foundations of biomedical ontology. In: Westerståhl D (Ed) Invited Papers from the 10th International Conference in Logic Methodology and Philosophy of Science (Oviedo, Spain, 2003). King's College Publications (London): 505-520.

Snodgrass RE (1941) The male genitalia of Hymenoptera. Smithsonian Miscellaneous Collections 99: 1-86.

Talamas E, Masner L, Johnson N (2011) Revision of the Paridris nephta species group (Hymenoptera, Platygastroidea, Platygastridae). ZooKeys 133: 49-94.

Vilhelmsen L (1996) The preoral cavity of lower Hymenoptera (Insecta): comparative morphology and phylogenetic significance. Zoologica Scripta 25: 143-170.

Vilhelmsen L, Mikó I, Krogmann L (2010) Beyond the wasp-waist: structural diversity and phylogenetic significance of the mesosoma in apocritan wasps (Insecta: Hymenoptera). Zoological Journal of the Linnean Society 159: 22-194.

Vogt L, Bartolomaeus T, Giribet G (2009) The linguistic problem of morphology: structure versus homology and the standardization of morphological data. Cladistics 26: 301-325. doi: 10.1111/j.1096-0031.2009.00286.x

Wagner GP (1989) The Biological Homology Concept. Annual Review of Ecology and Systematics 20: 51-69.

Wharton R, Cammack J, Mullins P (2010) A revision of the westwoodiine genus Pergaphaga (Hymenoptera, Ichneumonidae, Ctenopelmatinae). ZooKeys 37: 35-68.

Wiley EO, Lieberman BS (2011) Phylogenetics: Theory and Practice of Phylogenetic Systematics. Wiley-Blackwell, Hoboken.

Yoder MJ (2004) Revision of the North American species of the genus Entomacis (Hymenoptera: Diapriidae). The Canadian Entomologist 136: 323-405.

Yoder MJ (2007) Mannomicrus (Hymenoptera: Diapriidae), a new genus of myrmecophilic diapriid, with a digital version of Masner and Garcia’s (2002) key to New World Diapriinae and an illustration of digital description and key markup using an ontology. Zootaxa 1439: 47-55.

Yoder MJ, Mikó I, Seltmann KC, Bertone MA, Deans AR (2010) A gross anatomy ontology for Hymenoptera. PLoS ONE 5: e15991. doi: 10.1371/journal.pone.0015991 